\title{
Isolation of Frankia from the root nodules of Elaeagnus macrophylla and Alnus sieboldiana and their infectivities to the host plants
}

\author{
Hideo Sasakawa*, Hiroshi Kawai, \\ Norikatsu TaKahashi and SizUKa Tanigawa \\ Faculty of Agriculture, Okayama University, Okayama 700-8530, Japan
}

(Received January 23, 1998-Accepted July 1, 1998)

\begin{abstract}
We isolated endophytic Frankia from the root nodules of Elaeagnus macrophylla and Alnus sieboldiana and examined the infectivity of the isolates to the host plants.

One strain from E. macrophylla and 13 strains from A. sieboldiana were isolated by the simple method of suspending the nodule homogenate directly in liquid Qmod medium. Hyphae, sporangia and vesicles, which are morphological characteristics of Frankia, were observed in the E. macrophylla isolate. However, only hyphae and sporangia were observed in the $A$. sieboldiana isolate.

The E. macrophylla isolate formed nodules on the roots of the host plant and the average acetylene reduction activity (ARA) was $17.7 \pm 6.9 \mu \mathrm{mol} \mathrm{C}_{2} \mathrm{H}_{4} \mathrm{~h}^{-1}$ (g fresh wt nod.) ${ }^{-1}$. Eleven strains isolated from $A$. sieboldiana formed nodules on the root of the host plants, but 2 strains did not nodulate. The average ARA was $4.22 \pm 3.73 \mu \mathrm{mol} \mathrm{C}_{2} \mathrm{H}_{4} \mathrm{~h}^{-1}$ (g fresh wt nod.) ${ }^{-1}$.
\end{abstract}

Key words: actinorhizal plants, Frankia, infectivity, isolation, root nodules

\section{Introduction}

Most actinorhizal plants, which form nodules on their roots after infection with actinomycete Frankia, are capable of achieving higher rates of nitrogen fixation $^{4,5,9,14,15)}$ and the estimated amount of nitrogen fixed by those plants annually per unit area is comparable to that fixed by legumes ${ }^{8,10}$.

Actinorhizal plants can grow on barren soils deficient in nitrogen nutrients and some of them are used for the recovery of vegetation and improvement in the fertility of wastelands. For example, Alnus and Elaeagnus have been widely used for land reclamation of industrial wastelands, for stabilization, and for the

* Corresponding author; e-mail: sasakawa@ccews2.cc. okayama-u.ac.jp improvement of the fertility of degraded forest soils in Europe $^{2)}$. In Japan, Myrica rubra has been planted with pine trees in liparite soil in the Seto Inland Sea area of western Japan. The planting of Myrica rubra improved the soil $\mathrm{pH}$ and fertility, and promoted the growth of the pine trees ${ }^{17)}$. Alnus species such as $A$. firma and $A$. sieboldiana are commonly used as "fertilizer trees" to accelerate the growth of the target trees during forestation. Thus, introduction of actinozhizal plants is expected to be useful for the recovery of vegetation and fertility improvement in problem soils. For this purpose, it is necessary to select Frankia strains which have higher infectivity, forming many nodules with high nitrogen-fixing activity.

Here, we report the isolation of endophytic Frankia from the root nodules of E. macrophylla and A. sieboldiana, morphological characteristics of the isolated strains, their infectivity to the host plants, and the ni- 
trogen-fixing activity of the nodulated plants.

\section{Materials and Methods}

\section{Frankia isolation}

We previously isolated a Frankia strain from $M$. rubra nodules with different size mesh screens ${ }^{9}$. Here, we isolated Frankia from the root nodules of $E$. macrophylla and $A$. sieboldiana by a simple method. Nodules were thoroughly washed with running tap water to remove soil, rinsed three times with distilled water and then nodule lobes were cut with a razor blade. The lobes were surface-sterilized with Na-hypochlorite (available chlorine approximately $5 \%$ ) solution for $7 \mathrm{~min}$ and rinsed with distilled water 5 times. The lobes were further washed with $0.1 \%(\mathrm{v} / \mathrm{v})$ Tween 80 solution and sterilized water, and again surfacesterilized with $\mathrm{Na}$-hypochlorite solution. After being rinsed with sterilized water 5 times, each lobe was immersed in $0.15 \mathrm{~mL}$ of $50 \mathrm{mM}$ sodium phosphate buffer $(\mathrm{pH}=7.5)$ containing $1 \%(\mathrm{w} / \mathrm{v})$ polyvinyl pyrrolidone (K30)(Isizu Chemical Co.) for $15 \mathrm{~min}$ and ground with a glass rod in a test tube. The homogenate was then suspended directly in $3 \mathrm{~mL}$ of liquid Qmod medium ${ }^{11)}$ in another test tube and incubated at $28^{\circ} \mathrm{C}$ in the dark.

\section{Photomicrography}

An aliquot of isolated Frankia was transferred onto a slide glass and stained with one drop of $0.5 \%$ fuchsin (basic) solution for $1 \mathrm{~min}$ at room temperature. After putting a cover glass on the sample, distilled water was gently injected between the slide and the cover glass and excess dye was removed carefully with filter paper. The stained samples were observed with an Olympus photomicroscope.

\section{Inoculation test for host plants}

The seeds of $E$. macrophylla were germinated and grown in a mixture of autoclaved vermiculite and perlite (7:3) at room temperature. One 2-month-old seedling was transplanted into each plastic pot $(12 \mathrm{~cm}$ diameter, $10 \mathrm{~cm}$ depth) containing autoclaved vermiculite. A Frankia strain was propagated and collected as previously described ${ }^{13)}$ and diluted to 1,000 fold of packed cell volume with $\mathrm{N}$-free Arnon-
Hoagland solution ${ }^{1)}$. Twenty milliliters of the Frankia cell suspension was supplied, then the seedlings were cultured for 4 months at $25^{\circ} \mathrm{C}$ under artificial light conditions ( $\left.100 \mu \mathrm{E} \mathrm{m}^{-2} \mathrm{~s}^{-1}\right)$ and a day/ night regime of $14 \mathrm{~h} / 10 \mathrm{~h}$. N-free Arnon-Hoagland solution was supplied at one-week intervals.

About 100 A. sieboldiana seeds per pot were sown. The pots contained autoclaved vermiculite. The seeds were then cultured under artificial light conditions $\left(250 \mu \mathrm{E} \mathrm{m}^{-2} \mathrm{~s}^{-1}\right)$ and a day/night regime of $14 \mathrm{~h} / 10 \mathrm{~h}$ at $25^{\circ} \mathrm{C}$. Two weeks after germination, the seedlings were thinned to leave 10 vigorously growing seedlings per pot. They were then supplied $20 \mathrm{~mL}$ of a Frankia cell suspension diluted to 500 -fold of packed cell volume with $\mathrm{N}$-free Arnon-Hoagland solution. The seedlings were cultured for 2 months under the same conditions as described above. N-free Arnon-Hoagland solution was supplied at one-week intervals.

\section{Measurement of nitrogen-fixing activity}

Nitrogen-fixing activities were measured by the acetylene reduction method for intact plants in $E$. macrophylla and for nodulated roots in A. sieboldiana. The intact E. macrophylla seedling was fixed at the stem portion with greasy clay in a hole bored in the center of a rubber stopper and the root portion was introduced into a $200 \mathrm{~mL}$ glass bottle. The gap between the glass bottle and rubber stopper was sealed with greasy clay to prevent gas leakage. The nodulated roots of an $A$. sieboldiana were in a $110 \mathrm{ml}$ Erlenmeyer flask and sealed tightly with a rubber stopper. Gas phases in the containers were adjusted to contain 10\% acetylene and incubated at $25^{\circ} \mathrm{C}$ under room light conditions. After a $1 \mathrm{~h}$ incubation, the ethylene produced was measured as described previously ${ }^{14)}$.

\section{Results and Discussion}

\section{Isolation of Frankia}

Several hundred nodule lobes were used in the isolation of endophytic Frankia. Test tubes contaminated with bacteria or fungi were eliminated over the 10 days from the commencement of incubation. The percentage of such contaminated test tubes was approximately $40 \%$ to $50 \%$. The uncontaminated ones were fur- 
ther incubated. After a 1 month incubation of the nodule lobe homogenate, colonies of filamentous bacteria, which were clearly different from fungi, ap-

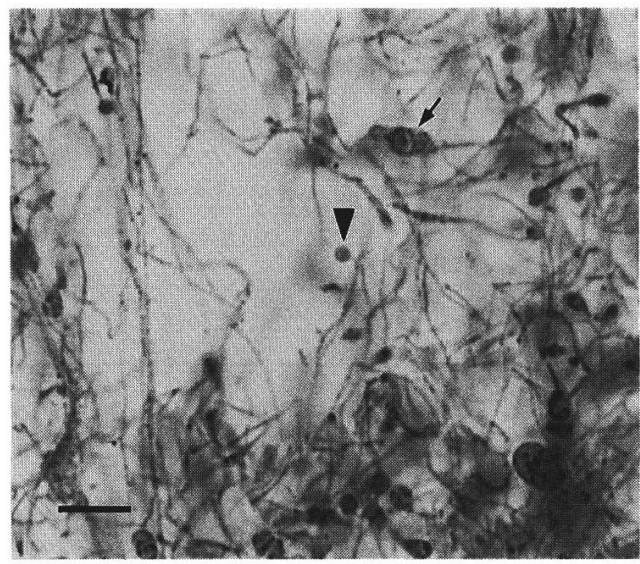

Fig. 1. Photomicrograph of the Frankia strain, Ema 1, isolated from root nodules of Elaeagnus macrophylla. Arrow $(\downarrow)$ and arrowhead $(\nabla)$ indicate the sporangium and the vesicle, respectively. Bar $=10 \mu \mathrm{m}$. peared in some test tubes. Under microscopical observation, 1 strain from E. macrophylla and 13 strains from $A$. sieboldiana were isolated. Hyphae, sporangia and vesicles which are morphological characteristics of Frankia were observed in the $E$. macrophylla isolates (Fig. 1). However, only hyphae and sporangia were observed in the $A$. sieboldiana isolates (Fig. 2). We designated the strain isolated from E. macrophylla as Ema1 and numbered the strains isolated from $A$. sieboldiana from As1 to As13. Although the formation of vesicles is an important parameter in identifying morphologically if the isolate is Frankia, some strains form vesicles only when they are cultured in a defined nutrient medium with a limited supply of nitrogenous compounds and express nitrogen-fixing activity ${ }^{3,9,16)}$. Fukumoto et al. ${ }^{5)}$ isolated an infective Frankia strain from $A$. sieboldiana and reported that only sporangia were observed microscopically. These findings suggest that Frankia from $A$. sieboldiana does not form or forms very few vesicles in pure culture.
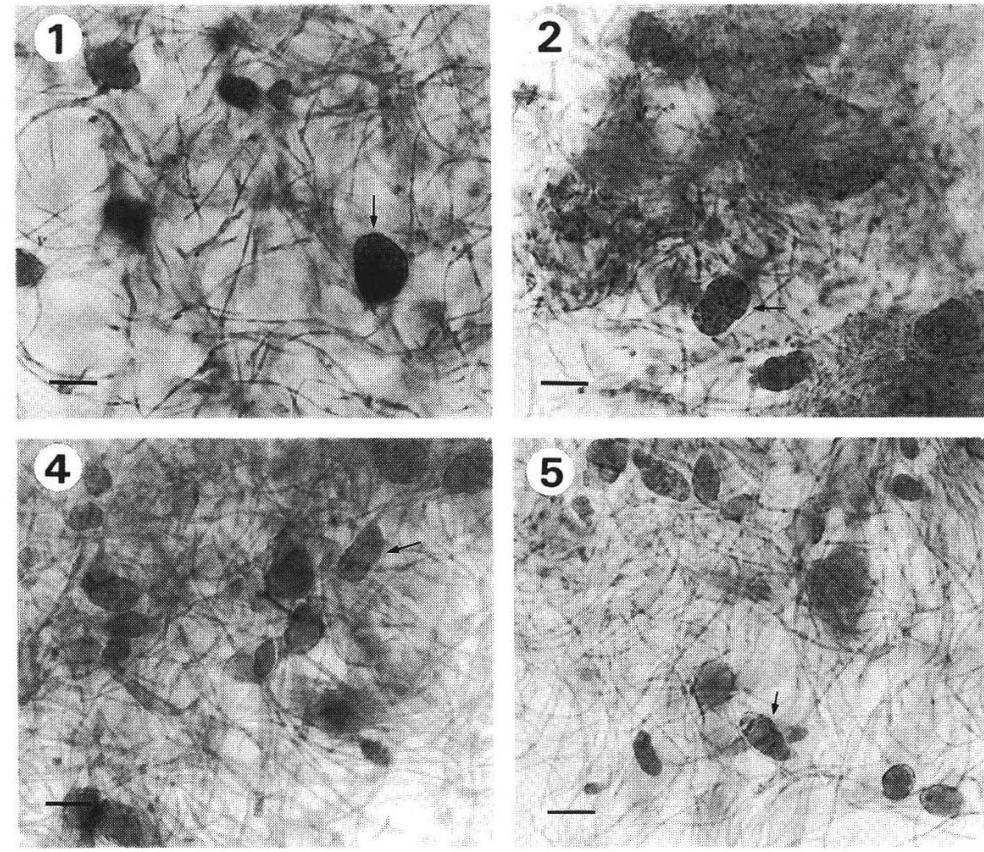

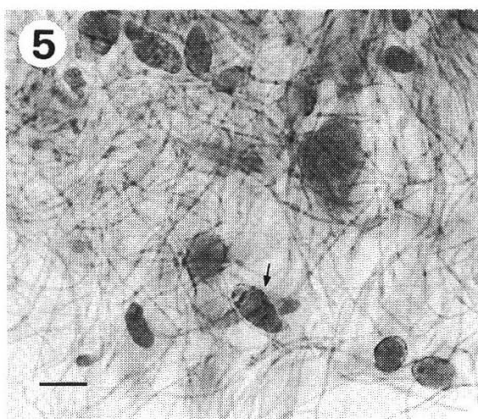

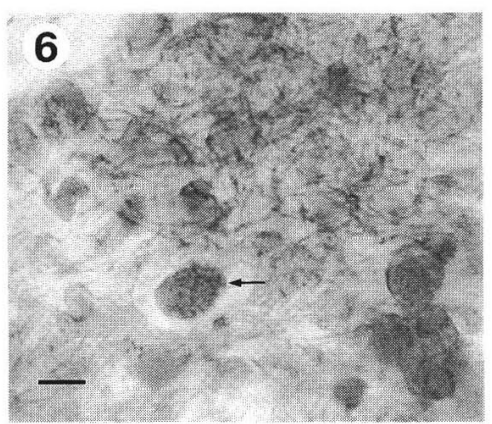

Fig. 2. Photomicrograph of Frankia strains isolated from root nodules of Alnus sieboldiana. Numbers 1 to 6 indicate strains As1 to As6 and arrows indicate sporangia. Bar $=10 \mu \mathrm{m}$. 

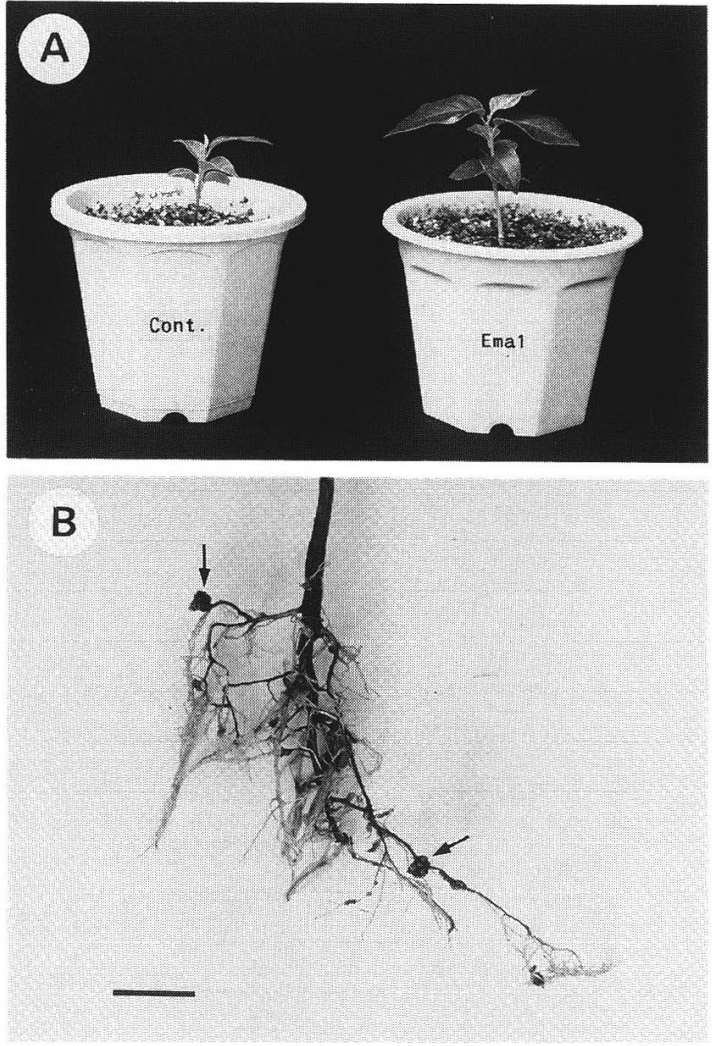

Fig. 3. Growth and nodules of Elaeagnus macrophylla formed by inoculation of Frankia strain Ema1. Fig. 3A shows the growth of plants inoculated with the Frankia strain Ema1. The left pot is an uninoculated control. Fig. 3B shows nodules formed on a plant. Plants were cultured for 4 months after inoculation. Arrows indicate nodules. $\mathrm{Bar}=10 \mathrm{~mm}$.

\section{Inoculation test}

Each isolate was inoculated onto host plants growing in vermiculite. The growth of inoculated $E$. macrophylla plants was more vigorous than that of uninoculated plants (Fig. 3A) and many nodules were formed on the roots of the seedlings inoculated with the Ema1 strain (Fig. 3B). The average ARA of 6 plants was $17.7 \pm 6.9 \mu \mathrm{mol} \mathrm{C}_{2} \mathrm{H}_{4} \mathrm{~h}^{-1}$ (g fresh wt nod.) ${ }^{-1}$ (Table 1). This value was 40 times higher than that of the nodules of Eleagnus pungens formed by inoculation of Frankia isolated from the nodules of the host plant ${ }^{7}$. This large difference in ARA between the nodules of $E$. macrophylla and $E$. pungens may be related to the age of the nodules used in the determination of ARA. However, there is a possibility that the Frankia strain we isolated is more infective and forms nodules with higher ARA activity than the strains isolated from E. pungens by Fukumoto et al. ${ }^{7}$. The Ema1 strain formed vesicles in pure culture (Fig. 1). In contrast, no vesicles were observed in pure culture of the Frankia strains from E. pungens ${ }^{7}$. The formation of vesicles in pure culture may also be related to the formation of nodules with higher ARA activity.

Figure 4 shows the growth of $A$. sieboldiana seedlings inoculated with 13 isolates and the nodulated plants. The growth of the seedlings was more vigorous after inoculation of the 11 strains, except for As3 and As7, than the uninoculated seedlings (Fig. 4A). Many root nodules were formed on the roots of seedlings showing active growth (Fig. 4B). Nodule formation, ARA, and shoot fresh weight after inocula-

Table 1. Acetylene-reducing activity of Elaeagnus macrophylla seedlings 6 months after inoculation with a Frankia strain Ema1

\begin{tabular}{c|ccc}
\hline plant & \multicolumn{2}{|c}{ ARA } & nodule fr wt \\
\hline & $\mu$ mol C $_{2} \mathrm{H}_{4} \mathrm{~h}^{-1}$ plant $^{-1}$ & $\mu \mathrm{mol} \mathrm{C}_{2} \mathrm{H}_{4} \mathrm{~h}^{-1}(\mathrm{~g} \text { fr wt nod })^{-1}$ & $\mathrm{mg}$ \\
2 & 1.12 & 11.3 & 99 \\
3 & 1.55 & 14.1 & 110 \\
4 & 0.96 & 24.6 & 39 \\
5 & 0.78 & 14.7 & 53 \\
6 & 0.73 & 28.1 & 26 \\
\hline \multirow{2}{*}{ av. \pm SD } & 1.07 & 13.5 & 79 \\
\hline
\end{tabular}



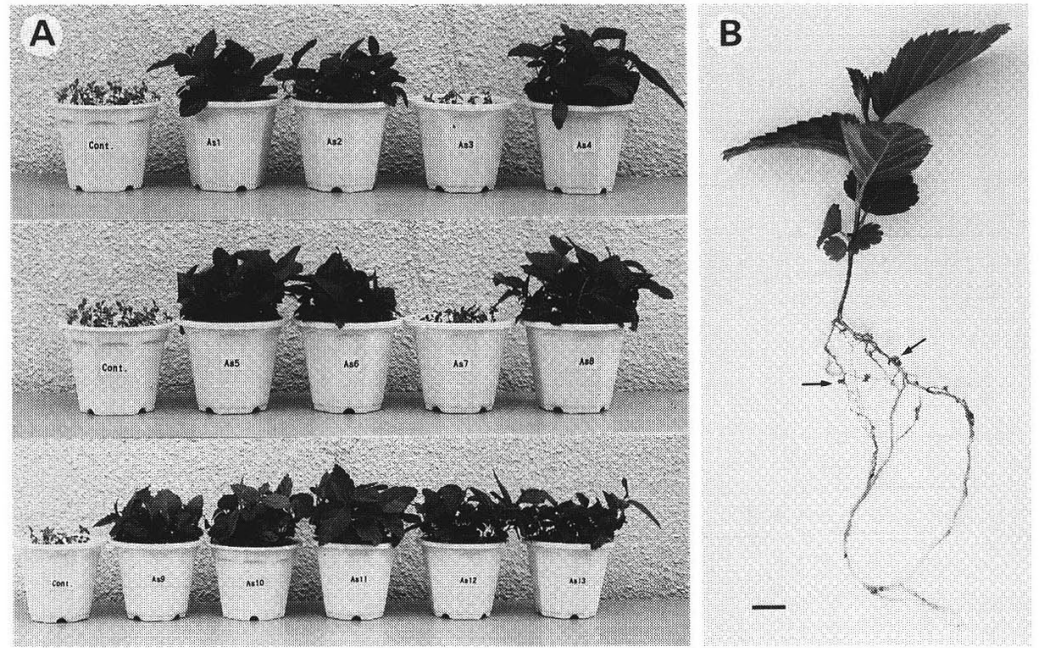

Fig. 4. Growth and nodules of Alnus sieboldiana formed by inoculation with Frankia strains. Fig. 4A shows the growth of plants inoculated with Frankia strains As1 to As 13. The left most pot is an uninoculated control. Fig. 4B shows nodules formed on a plant. Plants were cultured for 2 months after inoculation. Arrows indicate nodules. Bar $=10 \mathrm{~mm}$.

Table 2. Acetylene-reducing activity of Alnus sieboldiana seedlings 2 months after inoculation with Frankia strains

\begin{tabular}{l|ccccc}
\hline strain & nodule No. & nodule fr wt & \multicolumn{2}{c}{ ARA } & shoot fr wt \\
\hline & & $\mathrm{mg}$ & nmol $\mathrm{C}_{2} \mathrm{H}_{4} \mathrm{~h}^{-1} \mathrm{plant}^{-1}$ & $\mu \mathrm{mol}_{2} \mathrm{H}_{4} \mathrm{~h}^{-1}(\mathrm{~g} \text { fr wt nod })^{-1}$ & $\mathrm{~g}$ \\
As 1 & $19 \pm 6^{*}$ & $70 \pm 37$ & $186 \pm 94$ & $2.66 \pm 0.53$ & $1.461 \pm 0.545$ \\
As 2 & $23 \pm 4$ & $37 \pm 12$ & $71 \pm 64$ & $1.87 \pm 1.47$ & $0.908 \pm 0.218$ \\
As 3 & 0 & 0 & 0 & 0 & $0.028 \pm 0.007$ \\
As 4 & $37 \pm 16$ & $33 \pm 19$ & $183 \pm 164$ & $4.96 \pm 3.27$ & $0.748 \pm 0.361$ \\
As 5 & $43 \pm 12$ & $43 \pm 16$ & $329 \pm 296$ & $7.19 \pm 6.10$ & $1.005 \pm 0.338$ \\
As 6 & $33 \pm 4$ & $39 \pm 15$ & $302 \pm 498$ & $5.54 \pm 6.91$ & $1.041 \pm 0.173$ \\
As 7 & 0 & 0 & 0 & 0 & $0.035 \pm 0.016$ \\
As 8 & $57 \pm 25$ & $43 \pm 22$ & $110 \pm 53$ & $3.00 \pm 1.79$ & $0.962 \pm 0.380$ \\
As 9 & $65 \pm 21$ & $46 \pm 15$ & $179 \pm 135$ & $3.52 \pm 1.58$ & $0.731 \pm 0.179$ \\
As10 & $60 \pm 24$ & $42 \pm 21$ & $289 \pm 211$ & $6.72 \pm 2.77$ & $0.875 \pm 0.349$ \\
As11 & $70 \pm 27$ & $45 \pm 19$ & $179 \pm 94$ & $4.47 \pm 2.42$ & $1.141 \pm 0.407$ \\
As12 & $33 \pm 11$ & $29 \pm 15$ & $91 \pm 69$ & $2.88 \pm 1.40$ & $0.910 \pm 0.283$ \\
As13 & $30 \pm 10$ & $41 \pm 24$ & $125 \pm 74$ & $3.05 \pm 0.81$ & $0.821 \pm 0.420$ \\
\hline average & $43 \pm 24$ & $42 \pm 23$ & $187 \pm 223$ & $4.22 \pm 3.73$ & $0.890 \pm 0.451$ \\
\hline
\end{tabular}

* Numbers indicate average \pm SD of 7 plants.

tion of the 13 strains are summarized in Table 2. Eleven strains produced nodules on $A$. sieboldiana seedlings but 2 strains, As3 and As7, did not. The average ARA of the $A$. sieboldiana nodules was $4.22 \pm 3.73 \mu \mathrm{mol} \mathrm{C}_{2} \mathrm{H}_{4} \mathrm{~h}^{-1}$ (g fresh wt nod.) ${ }^{-1}$, which is equivalent to the value reported previously for $A$. sieboldian $a^{5,6)}$ and $M$. rubra ${ }^{13)}$. Judging from both nitrogen-fixing activity (ARA) shown in Table 2 and the growth rate in pure culture (data not shown), As5 and As10 are the most effective strains of $A$. sieboldiana.

Root nodules were not formed on the roots of the host plants after inoculation of the As 3 and As7 
strains. Lechevalier and Lechevalier ${ }^{12)}$ classified frankiae into two broad groups, A and B. Group A is very heterogeneous and contains strains that may not be infective to the host from which they were isolated and most of these strains grow rapidly. In contrast, Group B is far more homogeneous and strains in this group grow relatively slowly while being infective to their original host plants. Both As3 and As7 grew relatively rapidly compared to other strains isolated from A. sieboldiana (data not shown). Furthermore, the hyphae of As3 and As7 had greater diameters than those of other infective strains and the ability of sporangia formation was lost due to subculture (data not shown). Accordingly, they may be frankiae which lack infectivity to the host plant and can be classified as Group A or as other actinomycetes which differ from frankiae. Comparison of their characteristics, including DNA homology, against the infective Frankia strains is required to determine whether they are frankiae or not.

\section{Acknowledgments}

This work was supported by a grant (08 NP0901) from the Ministry of Education, Science, Sports, and Culture of Japan.

\section{References}

1) Arnon, D.I. and D.R. Hoagland. 1940. Crop production in artificial solutions and soils with special reference to factors influencing yield and absorption of inorganic nutrients. Soil Sci. 50: $463-471$

2) Baker, D.D. and C.R. Schwintzer, Introduction, in "The Biology of Frankia and Actinorhizal Plants", ed. by C.R. Schwintzer and J.D. Tjepkema. Academic Press, London, 1990, pp.1-13.

3) Burggraaf, A.J.P., and W.A. Shimpton. 1983. Studies on the growth of Frankia isolates in relation to infectivity and nitrogen fixation (acetylene reduction). Can. J. Bot. 61: 2774-2782.

4) Dilton, J.T. and D. Baker. 1982. Variation in nitrogenase activity among pure-cultured Frankia strains tested on actinorhizal plants as an induction of symbiotic compatibility. New Phytol. 92: 215-219.
5) Fukumoto, T., K. Ishizawa and N. Muto. 1992. A tube culture of Frankia plants for studying Frankiaroot interactions. Jpn. J. Soil Sci. Plant Nutr. 63: 325-331. (in Japanese with English summary).

6) Fukumoto, T., K. Ishizawa and N. Muto. 1994. Further characterization of Frankia sp. 13-3-2 and its inoculation tests onto Oobayashabushi (Alnus sieboldiana). Jpn. J. Soil Sci. Plant Nutr. 65: 392-399. (in Japanese with English summary).

7) Fukumoto, T., T. Wada and N. Muto. 1995. Purification and identification of Frankia isolated from Elaeagnus pungens root nodules. Jpn. J. Soil Sci. Plant Nutr. 66: 490-498 (in Japanese with English summary).

8) Hibbs, D.E. and K. Cromack, Jr. Actinorhizal plants in Pacific Northwest forest, in "The Biology of Frankia and Actinorhizal Plants", ed. by C.R. Schwintzer and J.D. Tjepkema. Academic Press, London, 1990, pp.343-363.

9) Hiyoshi, T., H. Sasakawa and M. Yatazawa. 1988. Isolation of Frankia strains from root nodules Myrica rubra. Soil Sci. Plant Nutr. 34: 107-116.

10) Kondo, M., and T. Yoneyama. 1990. Recent research progress in biological nitrogen fixation (26). Nitrogen fixation of field crops cultivated in various areas of the world (2). Agric. Hortic. 65: 863-870 (in Japanese).

11) Lalonde, M. and H.E. Calvert, Production of Frankia hyphae and spores as an infective inoculation for Alnus species, in "Symbiotic Nitrogen Fixation in the Management of Temperate Forests", ed. by J.C. Gordon, C.T. Wheeler and D.A. Perry, Oregon State University, Corvallis, Oreg, 1979, pp.95-110.

12) Lechevalier, M.P. and H.A. Lechevalier, Systematics, isolation, and culture of Frankia, in "The Biology of Frankia and Actinorhizal Plants", ed. by C.R. Schwintzer and J.D. Tjepkema. Academic Press, London, 1990, pp.35-60.

13) Sasakawa, H. 1995. Effect of Frankia inoculation on growth and nitrogen-fixing activity of Myrica rubra seedlings prepared aseptically. Soil Sci. Plant Nutr. 41: 691-698.

14) Sasakawa H, B.C. Trung and S. Yoshida. 1986. Stem nodulation on Aeschynomene indica plants by isolated rhizobia. Soil Sci. Plant Nutr. 32: 145-149.

15) Silvester, W.B. and S.L. Harris. 1989. Nodule structure and nitrogenase activity of Coriaria arborea in response to varying $\mathrm{pO}_{2}$. Plant Soil 118: 97-109.

16) Tjepkema, J.D., W. Ormerod and J.D. Torrey. 1980. Vesicle formation and acetylene reduction activity in Frankia sp. CPI1 cultured in defined nutrient media. Nature 287: 633- 635.

17) Uemura, S. 1971. Non-leguminous root nodules in Japan. Plant Soil, Special vol., pp.349-360. 